\title{
Holistic Link Quality Estimation-based Routing Metric for RPL Networks in Smart Grids
}

\author{
Sana Rekik, Nouha Baccour, Mohamed Jmaiel ReDCAD Laboratory \\ University of Sfax, Tunisia \\ \{sana.rekik, nouha.baccour, mohamed.jmaiel\}@redcad.org Khalil Drira \\ LAAS-CNRS, France \\ Univ. de Toulouse, France \\ khalil.drira@laas.fr
}

\begin{abstract}
Wireless Sensor Networks (WSNs) have been recognized as a promising communication technology for smart grid monitoring and control applications. Unfortunately, environmental conditions in smart grids are complex and harsh (electromagnetic interference, obstructions, fading, etc.), which turns radio links extremely unreliable. Routing protocols play a crucial role to overcome low-power link unreliability in smart grid environments. Especially, RPL (IPv6 Routing Protocol for Low Power and Lossy Networks) is an IETF standard that is supposed to meet the requirements of WSN-based smart grid communications. RPL routing metric relies on link quality estimation through ETX (Expected Transmission Count) assessment, to identify high quality links for data delivery. However, ETX is not sufficiently accurate as it assesses a particular link aspect, number of packet retransmissions over the link, and ignores other important aspects such as channel quality. Consequently, as confirmed by recent experimental studies, RPL can fail to identify routing paths with high quality links leading to high packet loss rates. In this paper, we propose an alternative routing metric for RPL based on holistic link quality estimation, where several link metrics are combined. Based on COOJA simulations, we demonstrate that our proposed routing metric improves RPL performance over traditional routing metrics, including the RPL default metric, mainly in terms of packet loss ratio, end-to-end delay, energy efficiency, and topology stability.
\end{abstract}

Keywords - Wireless sensor network, smart grid, link quality estimation, RPL routing. 


\section{INTRODUCTION}

Currently, we are witnessing the progressive migration of our existing electrical grids into smart grids. As a matter of fact, traditional electrical grids have several shortcomings calling for their modernization, namely (i.) the limited power resources and growing power demand, (ii.) the imbalance between power demand and supply, and (iii.) the lack of advanced communication, monitoring and diagnostic capabilities. Wireless Sensor Networks (WSNs) have been recognized as a promising communication technology for smart grid monitoring applications [1], [2]. They bring significant advantages compared with traditional wireless technologies, including rapid deployment, large areas coverage and low cost.

Unfortunately, low-power links (i.e., WSN radio links) in smart grid environments are shown to be extremely unreliable [3]. Their quality fluctuates over time and space, and connectivity is typically asymmetric. This is due to following: First, it is well known that WSN nodes use low cost and low-power radios, which turns communication links prone to noise, interference and multi-path distortions. Second, electric grid environments are typically characterized by highly corrosive conditions, e.g., rain, humidity, electric equipment's noise, electromagnetic interference, obstructions, vibrations, etc.

Routing protocols play a crucial role to overcome low-power link unreliability in smart grid environments, through efficient link quality estimation. Link quality-aware routing allows delivering data over paths composed of high quality links, which improves the end-to-end delivery rate and the communication reliability. Especially, RPL (IPv6 Routing Protocol for Low Power and Lossy Networks) [4] is an IETF standard protocol that is link quality-aware. Its routing metric is based on ETX (Expected Transmission Count) as a Link Quality Estimator (LQE). Generally, RPL has been argued to meet the requirements of WSN-based smart grid monitoring applications [5], [6].

The use of RPL networks (i.e., WSNs based on RPL for routing) in smart grid applications was investigated [6]-[8], focusing on AMI (Advanced Metering Infrastructure) applications. It was found that in large-scale deployments, some nodes in the RPL network select bad routing paths as they involve low quality links, which leads to low packet delivery rates [6], [7]. This RPL malfunctioning mainly pertains to the ETX metric, which was previously argued to be not sufficiently accurate [9]. Indeed, ETX does not provide a holistic link quality estimation as it 
assesses a particular link aspect, number of packet retransmissions over the link, and ignores other important aspects such as channel quality. On the other hand, Opt-FLQE (Optimized Fuzzy Link Quality Estimator) [10] is a recent LQE that was shown to overcome several traditional LQEs, especially ETX, in terms of reliability and reactivity. It provides a holistic link quality estimation through the combination of four link quality metrics using Fuzzy Logic, namely packet delivery, packet retransmissions, channel quality and link asymmetry.

The objective of this work is to ensure reliable end-to-end communication in RPL networks deployed in smart grids, through holistic link quality estimation. To achieve this goal, we propose an alternative routing metric for RPL based on Opt-FLQE that we call Opt-FLQE $E_{R M}$. Then, we compare its impact on the performance of RPL with that of representative routing metrics, including RPL default routing metric. Experimental results using COOJA simulator [11] show that Opt-FLQE $E_{R M}$ improves the end-to-end-delivery, reduces the communication delay, and also improves the energy efficiency, and the topology stability. The rest of this paper is organized as follows: In the next section, an overview of RPL routing protocol is given. In Section III, we discuss recent related works. In Section IV, we introduce our link-quality based routing metric for RPL. Performance analysis of the proposed metric are presented in Section V. We conclude in Section VI.

\section{RPL PROTOCOL: OVERVIEW}

RPL [4] is a tree routing standard recently designed for Low power and Lossy Networks such as WSNs. It is a proactive, distance vector and source routing protocol that mainly targets collection-based networks, characterized by multipoint-to-point traffic (i.e., traffic towards the root).

RPL establishes one or multiple Destination-Oriented Directed Acyclic Graphs (DODAGs) or simply DAGs, each one rooted towards a sink node denoted as DAG root. This root node can provide a default route to the Internet. The DAG structure slightly differs from a classical tree structure in the sense that a node can maintain a set of potential parents (instead of one) towards the destination node and then associates itself to a preferred parent.

The establishment and the maintenance of the DODAG are ensured by DIO (DODAG Information Object) control messages disseminated using a trickle timer. The DIO packets contain information such as the rank of the node (the relative distance of the node to the root), the 
Objective Function (OF) and the metrics used for path cost computation. The OF defines how the routing metrics are converted into a rank value. Two OFs have been specified by the ROLL working group: OF0 [12], which is a hop-count based metric, and Minimum Rank with Hysteresis Objective Function (MRHOF) [13], which uses ETX as a default routing metric.

\section{RELATED WORK}

Several routing metrics were proposed for RPL networks in order to satisfy application requirements, especially reliability ${ }^{1}$, delay and energy efficiency [14]-[16]. In [14], the authors considered both reliability and energy efficiency requirements and introduced two routing metrics. The first extends ETX by considering packet losses due to MAC contention, which improves the communication reliability. The second metric considers the minimal reliability required by the application and provides balanced power consumption among nodes by selecting the parent having the lightest traffic load. A routing metric that considers both energy and reliability requirements is proposed in [15], where reliability is considered using ETX. To account for three application requirements, namely reliability, delay and energy efficiency, the authors in [16] proposed the combination of several metrics: The remaining battery levels of the nodes, the ETX metric, the hop count and the end-to-end delay.

Differently from the above studies, the authors in [17], [18] addressed communication reliability in RPL networks by improving the link quality estimation process through sophisticated probing techniques. In [17], the authors targeted dense RPL networks where nodes have high number of neighbors. They proposed the use of passive probing instead of active probing. Passive probing is based on optimistic link quality estimation assuming that an unknown link is of good quality, i.e., having ETX equal to 1. This allows a node to keep switching the preferred parent among potentially good neighbors. On the other hand, the authors in [18] proposed the use of active probing at the network establishment phase, i.e., before data traffic begin to be exchanged. During this phase, broadcast probe packets are disseminated, using the trickle algorithm, allowing link quality estimation and thus network topology establishment. Once established, the nodes execute regular RPL, without the active probing mechanism.

All the research works described above share the following property: They tend to improve the communication reliability in RPL networks, where the reliability measurement over each

\footnotetext{
${ }^{1}$ Application reliability or communication reliability refers the end-to-end data delivery ratio
} 
individual link — the so called link quality estimation, is performed using RPL default metric: ETX.

ETX was initially introduced by Couto et al. [19], as the inverse of the product of the forward delivery ratio and the backward delivery ratio. It approximates the required number of packet transmissions/retransmissions over the link, before a successful reception. ETX was extensively used by the research community as a representative and reference LQE as well as a routing metric where the path cost is the sum of link estimates. However, ETX has several limitations: In [9], a set of representative LQEs including ETX, were experimentally evaluated using RadiaLE testbed [9]. It was found that ETX is not sufficiently accurate as it may over-estimate link quality. It is also not responsive to link quality degradation as it is computed at the receiver side. For instance, when the link is bad, packets are retransmitted many times without being successfully delivered to the receiver so that ETX can not be updated. The same observations was confirmed in [10], where ETX is evaluated in smart grid environments, characterized by highly corrosive conditions. Finally, the authors in [6], [7] evaluated RPL networks in smart grid applications, specifically in AMI applications. They found that in large-scale deployments, some nodes in the RPL network select bad routing paths as they involve low quality links, which leads to low packet delivery rates [6], [7]. This RPL malfunctioning mainly pertains to the ETX inaccuracy.

It is important to notice that ETX has many implementations. Although originally computed based on the inverse of packet delivery ratio [19], several other implementations compute ETX directly by counting the number of MAC retransmissions for a successful packet reception [20]. Nevertheless, we believe that all these implementations share a common limitation for ETX: They assess a particular link aspect, number of packet retransmissions over the link, and ignore several other important link aspects such as channel quality, link asymmetry etc. On the other hand, Opt-FLQE [10] (Optimized Fuzzy Link Quality Estimator — F-LQE [21]) is a recent link quality estimator that was shown to overcome several traditional LQEs such as ETX, in terms of reliability and reactivity. It combines four link metrics using Fuzzy Logic, namely packet delivery, packet retransmissions, channel quality and link asymmetry. Each metric captures a particular link aspect so that the overall link score provided by Opt-FLQE represents a holistic characterization of the link. Importantly, Opt-FLQE is specially designed for WSNs deployed in smart grid environments where links are shown to be extremely unreliable [3].

In summary, communication reliability in RPL networks is still an open research problem 
as ETX, the RPL default routing metric, is not sufficiently accurate. The need for an efficient routing metric based on holistic link quality estimation for RPL routing protocol is more than required, especially when RPL networks are deployed in harsh smart grid environments. In this paper, we devise using Opt-FLQE for the design of Opt-FLQE $E_{R M}$, an alternative routing metric for RPL, based on holistic link quality estimation.

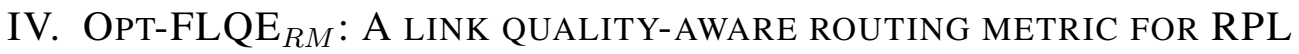

\section{A. Overview of Opt-FLQE}

Opt-FLQE estimates link quality on the basis of four link properties: (i.) packet delivery, assessed by the Smoothed Packet Reception Ratio (SPRR), (ii.) channel quality, assessed by the Average Signal-to-Noise Ratio (ASNR), (iii.) packet retransmissions, assessed by Smoothed Required Number of Packet retransmissions (SRNP), and (iv.) link asymmetry, assessed by the difference between the uplink PRR and the downlink PRR, noted as ASL (ASymmetry Level). Opt-FLQE provides a score ranging in [0..100], where 0 is the worst quality and 100 is the best.

Opt-FLQE considers each of these link properties as a fuzzy variable and combines them using Fuzzy Logic. The high quality of a link is characterized by the following fuzzy rule:

IF the link has high packet delivery AND low asymmetry AND low packet retransmissions AND high channel quality THEN it has high quality.

To produce a numerical value of the link quality (a score), the above rule translates to the following equation of the fuzzy measure of the link $i$ high quality.

$$
\begin{aligned}
\mu(i)=\beta \times \min \left(\mu_{S P R R}(i), \mu_{A S L}(i), \mu_{S R N P}(i), \mu_{A S N R}(i)\right) & \\
& +(1-\beta) \times \operatorname{mean}\left(\mu_{S P R R}(i), \mu_{A S L}(i), \mu_{S R N P}(i), \mu_{A S N R}(i)\right)
\end{aligned}
$$

The parameter $\beta$ is a constant set to 0.6 as recommended in [21]. $\mu_{S P R R}, \mu_{A S L}, \mu_{S R N P}$ and $\mu_{A S N R}$ represent membership functions in the fuzzy subsets of high packet reception ratio, low asymmetry, low packet retransmissions, and high channel quality, respectively. Each membership function has piecewise linear form, determined by two thresholds [10], [21].

Opt-FLQE is both a sender-side and a receiver-side estimator. When $w_{r}$ packets are received, a node computes $\mu_{S P R R}, \mu_{A S L}$ and $\mu_{A S N R}$ and then computes $\mu(i)$ based on the most recent value of $\mu_{S R N P}$. When $w_{t}$ packets are transmitted/re-transmitted, a node computes $\mu_{S R N P}$ and then updates $\mu(i)$. Finally, $\mu(i)$ values are smoothed using the EWMA filter, in order to provide 
stable link estimates. Opt-FLQE metric is then given by the following equation, where $\alpha$, equal to 0.9 as in [21], controls the smoothness:

$$
\operatorname{Opt}-\mathrm{FLQE}\left(\alpha, w_{r}, w_{t}\right)=\alpha \times \text { Opt-FLQE }+(1-\alpha) \times 100 \times \mu(i)
$$

In our implementation of Opt-FLQE in RPL, we made two modifications of Opt-FLQE. First, Opt-FLQE assesses channel quality using ASNR. In our implementation, we replace ASNR by the ARSSI (Average Received Signal Strength Indicator), which also allows channel quality assessment. This modification is justified by the fact that RSSI is more convenient to routing than SNR that involves more computation complexity and thus additional delay. RSSI is computed in one step, just by sampling the RSSI at packet reception. On the other hand, SNR is computed in three steps: (1) sampling the RSSI at packet reception to get the received signal (S), (2) sampling the RSSI just after packet reception to get the noise floor $(\mathrm{N})$, and (3) subtracting $\mathrm{N}$ from $\mathrm{S}$ to get SNR. Nevertheless, it is important to note that SNR is more accurate than RSSI in channel quality assessment.

Second, in our implementation of Opt-FLQE, we have omitted the ASL metric for the following reason: The combination of SRNP and SPRR provides a bidirectional link assessment and thus can capture link asymmetry property. In fact, based on several simulation runs, not included in this paper for the sake of brevity, we found that ASL metric does not contribute to a better link quality estimation (i.e., does not improve routing performance considerably). For this reason, we omit ASL metric for lower complexity and more energy efficiency.

\section{B. Opt-FLQE $E_{R M}$ :Opt-FLQE based Routing Metric}

Our objective is to use link quality estimates provided by Opt-FLQE to select optimal routing paths in terms of end-to-end reliability. MRHOF supports any additive routing metric and uses ETX by default. Thus, the path cost, interpreted as the total number of packet retransmissions over the path, is the sum of packet retransmissions of each link in this path. Opt-FLQE provides a score that does not refer to a physical phenomenon (e.g., packet retransmission or packet reception). To select optimal routing paths using Opt-FLQE, two potential candidate routing metrics are conceivable:

- $\sum$ Opt-FLQE ${ }_{i}$ : The sum of Opt-FLQE estimates of links composing the path. The path with the highest sum is selected for data delivery. 
- $\sum \frac{1}{\mathrm{Opt}_{\mathrm{FLQE}}}$ : The sum of inverse of Opt-FLQE of links constituting the path. The path with the minimum sum is selected.

To select the most efficient routing metric, we consider the following design requirements:

- An efficient link quality-based routing metric should achieve a trade-off between the path global quality and the quality of individual links composing the path. A path may have highest global quality among candidate paths; yet contains a weak quality link. This situation leads to several packet losses over this link, which negatively affects routing performance (e.g., the end-to-end packet delivery).

- An efficient routing metric should achieve a trade-off between the path length (number of hops) and the quality of individual links composing the path. Selecting short paths reduces the number of nodes involved for packet delivery, which conserves nodes energy and extends the network lifetime. However, a long path composed of good quality links may be better than a much shorter path with a low quality link.

Given the above design requirements, we believe that the second proposed metric $\left(\sum \frac{1}{\mathrm{Opt}_{\mathrm{FLQE}}}\right)$ is the most efficient for the following reasons: First, defining the link cost as $\frac{1}{\mathrm{Opt}_{-\mathrm{FLE}_{i}}}$ (instead of Opt-FLQE ${ }_{i}$ ) allows avoiding paths having low quality links: The poorer the link quality, the more costly it becomes, which impacts the overall path cost and reduces the probability of that path being selected. Second, the $\sum \frac{1}{\mathrm{Opt}^{-\mathrm{FLE}_{i}}}$ implicitly favors the selection of short paths: The longer the path (more hops), the more costly it becomes and the lower the chances of being selected. Hence, we define Opt-FLQE-based routing metric (Opt-FLQE $\left.{ }_{R M}\right)$, as follows:

$$
\text { Opt-FLQE }_{R M}(\text { Path }) \quad=\quad \sum_{i \in \text { Path }} \frac{1}{\text { Opt-FLQE }_{i}}
$$

$\frac{1}{\text { Opt-FLQE }_{i}}$ is the cost of link $i$ through Opt-FLQE computation. Thus, the path cost Opt-FLQE $E_{R M}$ is the sum of the link costs. The path having minimal cost is selected.

In what follows, we illustrate the above Opt-FLQE $R M$ features through an example in order to highlight its capability in selecting the most convenient path. Fig. 1 shows a network topology, where the source node $S$ has to select the most convenient path to reach the destination node $D$, giving Opt-FLQE estimates of all links. As shown in Fig. 1, there are three possible paths. The cost of these paths with respect to each routing metric is given in Table I. 


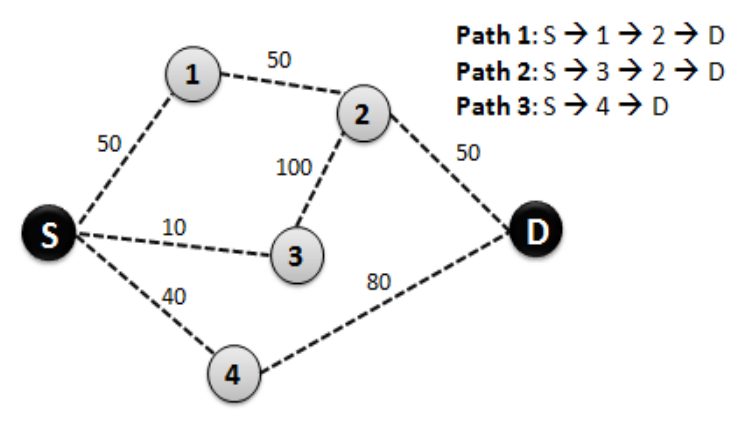

Fig. 1. An illustrative example to demonstrate the effectiveness of Opt-FLQE $E_{R M}$ metric.

TABLE I

PATH COST COMPUTATION ACCORDING TO DIFFERENT ROUTING METRICS.

\begin{tabular}{|ll|l|l|}
\hline & & $\sum$ Opt-FLQE $_{i}$ & $\sum \frac{1}{\text { Opt-FLQE }_{i}}$ \\
\hline $\begin{array}{l}\text { Path } \\
\text { cost }\end{array}$ & 1 & 150 & 0.06 \\
\hline $\begin{array}{l}\text { Path } \\
\text { cost }\end{array}$ & 2 & $\mathbf{1 6 0}$ & 0.13 \\
\hline $\begin{array}{l}\text { Path } \\
\text { cost }\end{array}$ & 3 & 120 & $\mathbf{0 . 0 3 7 5}$ \\
\hline
\end{tabular}

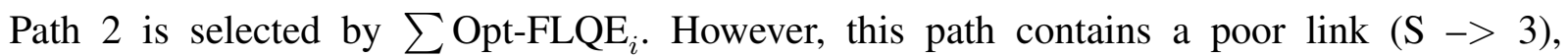
which will impact the overall path quality (e.g. in terms of delivery). Opt-FLQE $E_{R M}\left(\sum \frac{1}{\mathrm{Opt}^{-F_{L Q E}}}\right)$ chooses Path 3, which is indeed the most convenient path: It has the best global quality and the minimum hop count.

\section{Performance Analysis}

The objective of our simulation study is to demonstrate the potential of Opt-FLQE $E_{R M}$ in improving RPL routing performance, especially communication reliability. To this end, we propose to compare the impact of Opt-FLQE $E_{R M}$ on RPL performance, with that of ETX, the RPL routing metric, as well as four-bit [22], the CTP (Collection Tree Protocol) routing metric. Like ETX, four-bit approximates the required number of packet retransmissions. Basically, fourbit combines two metrics: RNP - a sender-initiated metric that counts the required number of packet retransmissions, and an ETX-like metric - a receiver initiated metric derived as the inverse of smoothed PRR. The two metrics are combined using the EWMA filter. 
We have integrated Opt-FLQE $R M$ and four-bit in ContikiRPL [20], the implementation of the RPL protocol in Contiki Operating System. The RPL routing protocol uses ETX as default routing metric. Hence, we have implemented two versions of RPL; in each version, we replace the default ETX by Opt-FLQE ${ }_{R M}$ and four-bit.

The performance evaluation considers the following metrics:

- Average packet loss ratio: It represents the ratio of the total number of lost packets (at the DAG root) to the total number of sent packets (by all RPL router nodes). This metric indicates the efficiency of a given routing metric in terms of end-to-end reliability, a key requirement for smart grid applications.

- Average end-to-end delay: It represents the average duration separating a packet transmission by the sender and its reception by the DAG root. This metric reflects the efficiency of a given routing metric in terms of communication delay, a key requirement for delay critical smart grid applications.

- Average hop count: It represents the average number of hops in a routing path.

- Average number of parent changes: It indicates the number of times a node has changed its parent. This metric reflects the topology stability induced by the used routing metric. Too many parent changes lead to unstable topology, but improve the quality of paths and thus the routing performance (e.g., packet reception ratio).

- Average power consumption: It represents the average consumed power by all nodes in the network. This metric reflects the efficiency of path selection. Typically, a good path (i.) contains minimum hops, which conserves energy by involving minimum number of nodes for data delivery, and (ii.) is constituted with high quality links, which also conserves energy by avoiding excessive packet retransmissions over bad quality links. Note that the radio communication is the greediest operation for a sensor node.

\section{A. Simulation settings}

To evaluate the impact of the considered routing metrics on RPL performance, we carried out simulations under COOJA [11], a well-known WSN emulator available as part of Contiki Operating System. We have considered a multi-hop network where nodes were deployed in a uniform grid topology. We varied the nodes number from 36 (i.e., $6 \times 6$ grid) to 100 (i.e., 10x10 grid). One node is set as sink node (i.e., DAG root) and the rest of the nodes act as router nodes. 
The sink node is located at coordinates $(0,0)$ and the grid unit is equal to 30 meters. All the nodes in Cooja were configured as Tmote Sky motes (with a CC2420 radio).

Each RPL router node periodically generates and sends data packets with a rate of 1 packet per minute. To enable the establishment of the topology, the nodes begin the transmission of data packets after a delay of 60 second (during this time, nodes exchange only beacon traffic). Each simulation run lasts 1 hour to ensure convergence to a steady state. Simulation results are provided with a $90 \%$ of confidence interval.

\section{B. Simulation results}

In this section, we compare the performance of Opt-FLQE $E_{R M}$ metric against the performance of ETX and four-bit, by analyzing their impact on RPL routing protocol. Comparisons are carried out in terms of communication reliability, delay, routing stability and energy efficiency. Numerical results are presented next.

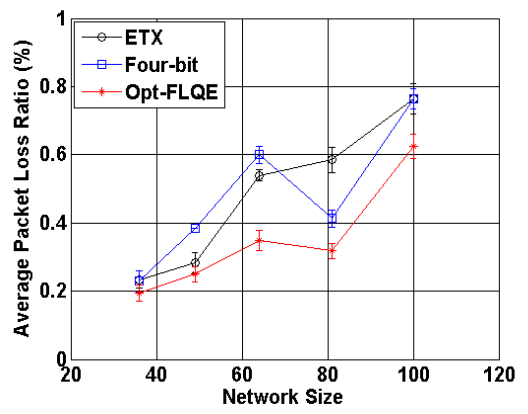

(a)

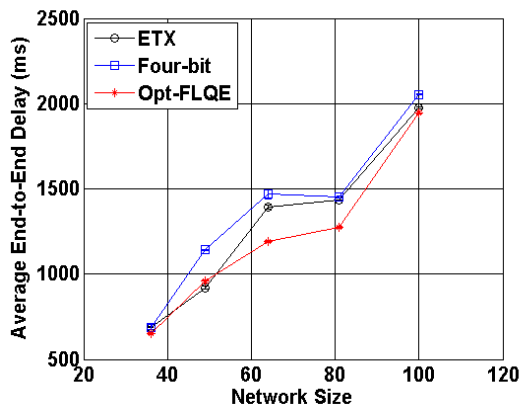

(b)

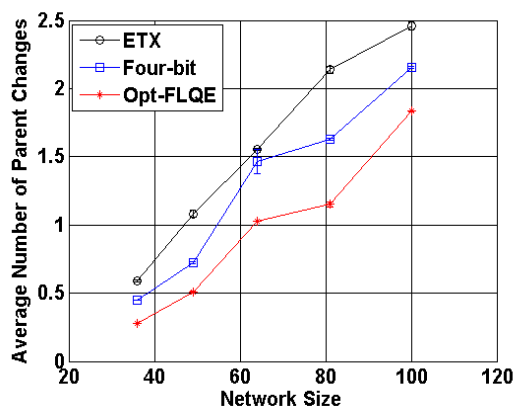

(c)

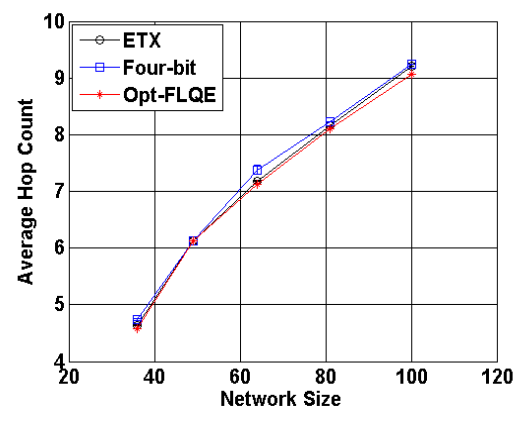

(d)

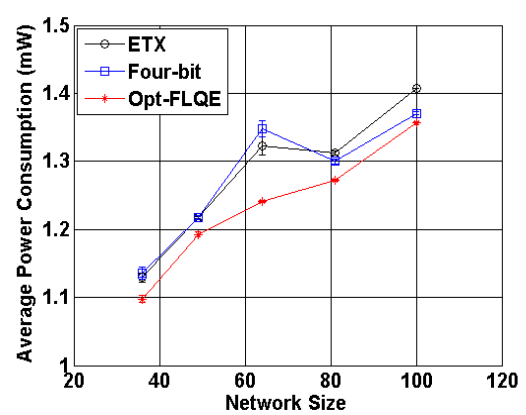

(e)

Fig. 2. Performance comparison of ETX, four-bit and Opt-FLQE as a function of nodes number. 
1) Communication reliability: Fig. 2a shows the average packet loss ratio of Opt-FLQE ${ }_{R M}$, four-bit and ETX. It is clear that Opt-FLQE $E_{R M}$ presents the lowest packet loss, which demonstrates communication reliability of the Opt-FLQE $E_{R M}$-based RPL network. These results can be justified by the accuracy of Opt-FLQE as a holistic link quality estimator as well as the efficiency of Opt-FLQE $R M$ as a routing metric. Routing based on Opt-FLQE allows identifying high quality links for data delivery. According to Opt-FLQE definition, such links are characterized by high packet delivery ratios, low number of packet retransmissions, and high channel qualities (refer to section IV-A). Combining these link features together into a link score to assess link quality leads to a better classification of links and allows picking up best quality links for routing path establishment. Further, the path cost evaluation in Opt-FLQE ${ }_{R M}$ also contributes to communication reliability, by avoiding paths having a low quality link.

2) Communication delay: Fig. 2b compares the average end-to-end delay of the three routing metrics: Opt-FLQE ${ }_{R M}$, four-bit and ETX. Despite the fact that the paths selected by the three metrics have almost the same length (refer to Fig. 2d), Opt-FLQE $E_{R M}$ has the lowest average endto-end delay. This result can be interpreted by the quality of paths selected by Opt-FLQE $E_{R M}$. One cause of communication delay is packet retransmissions. Choosing links involving low packet retransmissions, a property captured by Opt-FLQE, will then contribute to low communication delays.

3) Routing stability: Fig. 2c shows the average number of parent changes of Opt-FLQE ${ }_{R M}$, four-bit and ETX. It is clear that Opt-FLQE ${ }_{R M}$ has the lowest number of parent changes, which reflects the topology stability. This figure shows also that ETX is the most unstable metric as it leads to the highest parent changes. This is due to the following: In ContikiRPL implementation, ETX is computed based on data traffic. At the network establishment phase, only DIO messages are exchanged, and thus ETX link estimates are available only after the topology establishment, when data traffic is transmitted. Consequently, to join the DODAG, a node selects its parent arbitrarily. The network topology is then refined over time, and it converges to the optimal topology quite slowly. On the other hand, Opt-FLQE ${ }_{R M}$ (and four-bit as well) are computed based on both data and control (DIOs) traffic. Hence, the topology is established based on link quality information. Consequently, the selection of the node's parent at the topology establishment has a sense and the convergence to the optimal topology is faster. 
4) Energy efficiency: Fig. 2e depicts the average power consumption of the three evaluated routing metrics. From this figure, we can see that Opt-FLQE based routing metric leads to slightly lower power consumption. As explained previously, Opt-FLQE $E_{R M}$ allows minimizing packet loss (Fig. 2a) and therefore minimizing the number of retransmissions and maximizing the network lifetime.

In summary, simulation results presented in this section show that Opt-FLQE $E_{R M}$ improves RPL performance over ETX (the default routing metric of RPL) and four-bit. It efficiently establishes and maintains the network topology while ensuring (i.) low packet loss ratio, (ii.) low end-to-end delay, (iii.) low power consumption and (iv.) stable topology.

\section{CONCLUSION}

In this paper, we have presented a novel routing metric for RPL networks deployed in smart grids. The objective of the proposed metric is to ensure reliable end-to-end delivery in smart grid environments through efficient link quality estimation. In contrast to ETX, the default RPL routing metric that can only assesses a single link property (packet retransmissions), our proposed metric is based on holistic link quality estimation. It combines three link properties, namely packet retransmissions, packet delivery and channel quality. The proposed metric was evaluated through COOJA simulations, demonstrating its outperformance over ETX and fourbit, two representative routing metrics, in terms of packet loss ratio, end-to-end delay, energy efficiency, and topology stability.

\section{REFERENCES}

[1] M. Erol-Kantarci and H. T. Mouftah, "Wireless multimedia sensor and actor networks for the next generation power grid," Ad Hoc Networks, vol. 9, no. 4, pp. 542-551, 2011.

[2] E. Fadel, V. Gungor, L. Nassef, N. Akkari, M. A. Maik, S. Almasri, and I. F. Akyildiz, "A survey on wireless sensor networks for smart grid," Computer Communications, pp. -, 2015.

[3] V. C. Gungor, B. Lu, and G. P. Hancke, "Opportunities and challenges of wireless sensor networks in smart grid," IEEE Transactions on Industrial Electronics, vol. 57, no. 10, pp. 3557-3564, 2010.

[4] T. Winter, "Rpl: Ipv6 routing protocol for low-power and lossy networks," 2012.

[5] J. Hui, D. Popa, R. Salazar, N. Dejean, and J. Jetcheva, "Applicability statement for the routing protocol for low power and lossy networks (rpl) in ami networks," 2011.

[6] E. Ancillotti, R. Bruno, and M. Conti, "The role of the RPL routing protocol for smart grid communications," IEEE Communications Magazine, vol. 51, no. 1, pp. 75-83, 2013. 
[7] — _ "RPL routing protocol in advanced metering infrastructures: An analysis of the unreliability problems," in Sustainable Internet and ICT for Sustainability, SustainIT 2012, 4-5 October, 2012, Pisa, Italy, Sponsored by the IFIP TC6 WG 6.3 "Performance of Communication Systems", 2012, pp. 1-10.

[8] G. Rajalingham, Y. Gao, Q. Ho, and T. Le-Ngoc, "Quality of service differentiation for smart grid neighbor area networks through multiple RPL instances," in Q2SWinet'14, Proceedings of the 10th ACM Symposium on QoS and Security for Wireless and Mobile Networks, Montreal, QC, Canada, September 21-22, 2014, 2014, pp. 17-24.

[9] N. Baccour, A. Koubaa, M. B. Jamâa, D. do Rosário, H. Youssef, M. Alves, and L. B. Becker, "Radiale: A framework for designing and assessing link quality estimators in wireless sensor networks," Ad Hoc Networks, vol. 9, no. 7, pp. 1165-1185, 2011.

[10] S. Rekik, N. Baccour, M. Jmaiel, and K. Drira, "Low-Power link quality estimation in smart grid environments," in $I W C M C$ 2015 Wireless Sensor Symposium (IWCMC 2015 Wireless Sensor Symposium), Dubrovnik, Croatia, Aug. 2015.

[11] F. Osterlind, A. Dunkels, J. Eriksson, N. Finne, and T. Voigt, "Cross-level sensor network simulation with cooja," in Local Computer Networks, Proceedings 2006 31st IEEE Conference on, Nov 2006, pp. 641-648.

[12] P. Thubert, "Objective function zero for the routing protocol for low-power and lossy networks (rpl)," 2012.

[13] O. Gnawali, "The minimum rank with hysteresis objective function," 2012.

[14] P. D. Marco, C. Fischione, G. Athanasiou, and P. Mekikis, "Mac-aware routing metrics for low power and lossy networks," in Proceedings of the IEEE INFOCOM 2013, Turin, Italy, April 14-19, 2013, 2013, pp. 13-14.

[15] S. Capone, R. Brama, N. Accettura, D. Striccoli, and G. Boggia, "An energy efficient and reliable composite metric for RPL organized networks," in 12th IEEE International Conference on Embedded and Ubiquitous Computing, EUC 2014, Milano, Italy, August 26-28, 2014, 2014, pp. 178-184.

[16] O. Gaddour, A. Koubâa, and M. Abid, "Quality-of-service aware routing for static and mobile ipv6-based low-power and lossy sensor networks using RPL,” Ad Hoc Networks, vol. 33, pp. 233-256, 2015.

[17] S. Dawans, S. Duquennoy, and O. Bonaventure, "On link estimation in dense RPL deployments," in 37th Annual IEEE Conference on Local Computer Networks, Workshop Proceedings, Clearwater Beach, FL, USA, October 22-25, 2012, 2012, pp. 952-955.

[18] E. Ancillotti, R. Bruno, M. Conti, E. Mingozzi, and C. Vallati, "Trickle-1²: Lightweight link quality estimation through trickle in RPL networks," in Proceeding of IEEE International Symposium on a World of Wireless, Mobile and Multimedia Networks, WoWMoM 2014, Sydney, Australia, June 19, 2014, 2014, pp. 1-9.

[19] D. S. J. D. Couto, D. Aguayo, J. C. Bicket, and R. Morris, "a high-throughput path metric for multi-hop wireless routing," Wireless Networks, vol. 11, no. 4, pp. 419-434, 2005.

[20] N. Tsiftes, J. Eriksson, and A. Dunkels, "Low-power wireless ipv6 routing with contikirpl," in Proceedings of the 9th ACM/IEEE International Conference on Information Processing in Sensor Networks, ser. IPSN '10. New York, NY, USA: ACM, 2010, pp. 406-407.

[21] N. Baccour, A. Koubaa, H. Youssef, M. B. Jamâa, D. do Rosário, M. Alves, and L. B. Becker, "F-lqe: A fuzzy link quality estimator for wireless sensor networks," in EWSN, 2010, pp. 240-255.

[22] R. Fonseca, O. Gnawali, K. Jamieson, and P. Levis, "Four-bit wireless link estimation," in 6th ACM Workshop on Hot Topics in Networks - HotNets-VI, Atlanta, Georgia, USA, November 14-15, 2007. 